\title{
Centre du vaisseau USSMV: la voie trilatérale de la Suisse
}

\author{
En 2007, I'Union des Sociétés Suisses des Maladies Vasculaires (USSMV) a repris \\ l'idée d'un centre du vaisseau commun. En 2011, cette idée a été approuvée par les \\ comités de toutes les sociétés de discipline et par le comité de l'USSMV, et début \\ 2014, les 6 premiers centres ont été accrédités. Trois spécialistes des disciplines de \\ l'angiologie, de la chirurgie vasculaire et de la radiologie vasculaire intervention- \\ nelle se tiennent à la disposition des patients au centre du vaisseau $24 \mathrm{~h}$ sur 24 .
}

\section{Beat Frauchigera, \\ Stefan Küpferb, \\ Paolo Cassinac, \\ Lorenz Gürked, \\ Erik Haeslere, \\ Salah D. Qanadli ${ }^{\prime}$}

a Prof. Dr, président du Board centre du vaisseau USSMV (Union des Sociétés Suisses des Maladies. Vasculaires) Clinique médicale, hôpital cantonal de Frauenfeld

b Dr, président de l'USSMV, Venenzentrum Bad Ragaz

c PD Dr, membre du Board du centre du vaisseau USSMV, délégué SSP, cabinet de chirurgie et de chirurgie vasculaire, Lugano

d Prof. Dr, membre du Board du centre du vaisseau USSMV, délégué SSCV, chirurgie vasculaire, hôpital universitaire de Bâle

e Dr, membre du Board du centre du vaisseau USSMV, délégué SSA, cabinet d'angiologie, Morges

f Prof. Dr, membre du Board du centre du vaisseau USSMV, délégué SSCVIR, Service Radiodiagnostique et Radiologie interventionnelle, CHUV, Lausanne

Correspondance:

Prof. Dr Beat Frauchiger

Médecin-chef Clinique médicale

Hôpital cantonal

CH-8500 Frauenfeld

beat.frauchiger[at]stgag.ch

\section{Antécédents}

La médecine vasculaire moderne est un domaine interdisciplinaire par excellence dans la pratique hospitalière quotidienne. Le diagnostic et le traitement des maladies artérielles, des veines ou des vaisseaux lymphatiques nécessitent, selon la situation clinique, un travail technique important avec les exigences d'infrastructures et de personnel correspondantes. De plus, s'agissant des troubles de la circulation artérielle, les patients sont généralement polymorbides. Une évaluation globale correcte, une évaluation pronostique de l'évolution et le choix sur mesure de la procédure qui convient sont d'une haute importance clinique. Aucun des spécialistes impliqués généralement dans le traitement des maladies vasculaires, qu'il s'agisse de l'angiologue, du chirurgien vasculaire ou du radiologue interventionnel, n'est en mesure, à lui seul, de couvrir l'ensemble des besoins du patient souffrant d'une maladie vasculaire. Contrairement à ce qui se passe dans d'autres pays, notamment anglo-saxons, où les spécialistes se déclarent compétents pour tout le spectre de traitements, l'idée d'une collaboration interdisciplinaire optimale s'est imposée de manière précoce en Suisse. Dans le contexte de cette interdisciplinarité qui a déjà fait ses preuves au quotidien, la Société Suisse d'Angiologie SSA et la Société Suisse de Phlébologie SSP ont fusionné en 1987 pour créer l'Union des Sociétés Suisses des Maladies Vasculaires (USSMV). En 2001, la Société Suisse de Chirurgie Vasculaire SSCV, la Société Suisse de Radiologie Vasculaire Interventionnelle SSCVIR et la Société Suisse de Microcirculation et de recherche vasculaire ont rejoint l'Union. Celle-ci est aujourd'hui le forum de médecine vasculaire le plus important du pays, et réalise en alternance annuelle le congrès commun de l'USSMV.

\section{Concept du centre du vaisseau}

Cette alliance politique des intérêts de la branche médicale vasculaire et la collaboration vécue déjà en divers endroits de manière exemplaire ont été la base du concept repris en 2007 de création d'un centre du vaisseau USSMV. Diverses étapes ont permis aux spécialistes des différentes sociétés de discipline médicale d'élaborer un concept de base pour l'établissement d'un label commun de médecine vasculaire interdisciplinaire. Ce concept a été approuvé en 2011 par les comités de toutes les sociétés de discipline et le comité de l'Union des Sociétés Suisses des Maladies Vasculaires. Ensuite, diverses étapes de projet ont permis d'élaborer une liste de critères concrets permettant d'évaluer les candidats au label de centre du vaisseau USSMV. Le catalogue de critères (Tableau 1) a été approuvé par toutes les sociétés et le comité de l’USSMV mi-2012.

\section{Mise en œuvre}

Le comité de l'USSMV a nommé un conseil (board) comme organe exécutif pour la mise en œuvre de la procédure de certification. Celui-ci se compose d'un président et d'un délégué de chaque société de discipline médicale (angiologie, chirurgie vasculaire, phlébologie et radiologie interventionnelle vasculaire). Par la suite, le board a concrétisé la liste de critères établie, et élaboré l'organisation nécessaire à la procédure de certification. Début 2013, la procédure de certification a été ouverte par un «call for centers». Dans une première étape, les centres candidats devaient établir un dossier pour documenter la mé-

\section{Les membres de I'USSMV}

(Union des Sociétés Suisses des Maladies Vasculaires)

- SSA: Société Suisse d'Angiologie, présidente Prof. Dr Béatrice Amann

- SSCV: Société Suisse de Chirurgie Vasculaire, président Prof. Dr Lorenz Gürke

- SSCVIR: Société Suisse de Radiologie Cardiovasculaire et Interventionnelle Prof. Dr. Salah D. Qanadli

- SSP: Société Suisse de Phlébologie, présidente PD Dr méd. Christina Jeanneret

- SSVMR: Société Suisse de Microcirculation et de recherche vasculaire, présidente Prof. Dr rer. physiol. Britta Engelhardt 
Tableau 1

Catalogue des critères centre du vaisseau USSMV.

\begin{tabular}{|c|c|c|c|}
\hline $\mathrm{N}^{\circ}$ & Critère & Etat/explication & Dur/souple \\
\hline 1. & Personnel & $\begin{array}{l}1 \text { Chirurgien vasculaire } \\
1 \text { Angiologue } \\
1 \text { Radiologue }\end{array}$ & $\mathrm{D}$ \\
\hline 2. & Coordination du CV & en alternance un coordinateur & $\mathrm{D}$ \\
\hline 3. & Déroulements au CV & Processus consignés par écrit & $\mathrm{D}$ \\
\hline 4. & Egalité des droits & cf. point 3 & $\mathrm{D}$ \\
\hline 5. & Colloque vasculaire & $\begin{array}{l}\text { Mini 1/semaine discussions de cas } \\
\text { Consignation par écrit des } \\
\text { décisions, résultats et complications } \\
\text { Décisions si possible en présence } \\
\text { des } 3 \text { partenaires }\end{array}$ & D \\
\hline 6. & Service 24 h sur 24 & $\begin{array}{l}\text { Pour diagnostic non invasif, } \\
\text { interventions par cathéter } \\
\text { périphérique et opérations } \\
\text { vasculaires }\end{array}$ & $\mathrm{D}$ \\
\hline 7. & Stratégie Processus patients & $\begin{array}{l}\text { Description/algorithme de } \\
\text { l'itinéraire d'un patient vasculaire, } \\
\text { y compris contrôles de suivi }\end{array}$ & $\mathrm{D}$ \\
\hline 8. & Itinéraires de patients & Minimum 6 itinéraires définis & $\mathrm{D}$ \\
\hline 9. & Contrôles de suivi & $\begin{array}{l}\text { Approprié, objectif, collégial (s) } \\
\text { Tous les CS documentés (h) } \\
\text { Peut être consulté en tout temps } \\
\text { par tous les partenaires (d) } \\
\text { Information réciproque active en } \\
\text { cas d'évolutions inattendues (d) }\end{array}$ & D-S \\
\hline 10. & Consultation plaies & & $\mathrm{D}$ \\
\hline 11. & Travail de prévention & Fixé dans le guide & $\mathrm{S}$ \\
\hline 12. & Réhabilitation MAOP & & $\mathrm{S}$ \\
\hline 13. & USI/Intermed. Care & & $\mathrm{D}$ \\
\hline 14. & Radiologie & & $\mathrm{D}$ \\
\hline 15. & Parc d'appareils non invasifs & & $\mathrm{D}$ \\
\hline 16. & $\begin{array}{l}\text { Traitement compressif et drainage } \\
\text { lymphatique }\end{array}$ & & $S$ \\
\hline 17. & Assurance-qualité interne & & $\mathrm{D}$ \\
\hline 18. & Assurance-qualité externe & & $\mathrm{D}$ \\
\hline 19. & $\begin{array}{l}\text { Etablissements de formation } \\
\text { postgraduée }\end{array}$ & & $\mathrm{S}$ \\
\hline 20. & Perfectionnement interdisciplinaire & & $S$ \\
\hline 21. & Recherche clinique & & $S$ \\
\hline 22. & $\begin{array}{l}\text { Passerelles pour formations continues } \\
\text { à des aptitudes spéciales }\end{array}$ & & $\mathrm{S}$ \\
\hline 23. & Ambiance au centre du vaisseau & $\begin{array}{l}\text { atmosphère ouverte, collégiale, } \\
\text { serviable, pas d'exclusions }\end{array}$ & $\mathrm{S}$ \\
\hline
\end{tabular}

Définitions/abréviations: $\mathrm{CV}$ = centre du vaisseau; $\mathrm{D}=$ critère dur: obligatoire; $\mathrm{S}$ = critère souple: souhaitable mais pas obligatoire cations. Mi-février 2014, les six premiers centres étaient officiellement accrédités. Le tableau 2 présente la liste des institutions accréditées fin juillet 2014.

\section{Tableau 2}

Liste des centres vasculaires USSMV déjà accrédits, état fin juillet 2014.

\author{
Hôpital cantonal de Bâle-Campagne, site Bruderholz \\ Spital Thurgau AG, site Frauenfeld \\ Hôpital cantonal de Bâle campagne, site Liestal \\ VASC AG Berne, site hôpital du Lindenhof \\ Hôpital du Valais, Sion \\ Hôpital de Thoune-Simmental, site Thoune \\ Hôpital universitaire de Zurich
}

\section{Caractéristiques principales du centre du vaisseau USSMV}

Le tableau 1, Liste des critères, présente des points importants qui doivent être respectés pour une certification. Au centre, il y a le contrôle de la collaboration optimale des trois spécialistes des vaisseaux (angiologie, chirurgie vasculaire et radiologie interventionnelle vasculaire). Les paramètres de la structure et des processus doivent ainsi être consignés. Les paramètres de résultats et les chiffres ne sont pas essentiels pour la certification d'un centre du vaisseau USSMV. S'agissant des paramètres de résultats, il existe certaines réserves fondamentales. Les chiffres de cas peuvent exclure de la certification les petites équipes qui fonctionnent bien et fournissent un travail quotidien de grande qualité, ce que l'USSMV veut justement éviter. En principe, il faut admettre que dans une institution où l'angiologie, la chirurgie vasculaire et la radiologie interventionnelle vasculaire collaborent on atteindra un nombre important de cas dans divers segments. Des procédures rares qui impliquent un travail nécessitant beaucoup de personnel et de matériel sont généralement concentrées sur les centres universitaires.

\section{Force}

L'atout principal du centre du vaisseau USSMV est l'ampleur du spectre diagnostique et thérapeutique de ce type d'institutions. De nombreux patients sont vus, parfois même sans facture supplémentaire, par les trois spécialistes durant la procédure de diagnostic et de traitement. Ces derniers ont, même s'ils ne mènent pas le traitement, des diagnostics différentiels et des idées supplémentaires en matière de traitement. L'évaluation globale apporte une deuxième et une troisième opinion à peu de frais sur le plan de traitement. Le principe du triple contrôle protège le patient de décisions thérapeutiques autistes ou de présentations enjolivées des résultats d'interventions. La disponibilité $24 \mathrm{~h}$ sur 24 et 365 jours par an des trois spécialistes des vaisseaux constitue égale- 
ment un élément important de la qualité de la structure. Au centre du vaisseau USSMV, le patient souffrant de maladie vasculaire peut compter jour et nuit sur une évaluation compétente, même de plusieurs spécialistes en cas de besoin. laire pratiquée également depuis toujours en dehors des centres, dans de petites structures et notamment aussi dans le secteur ambulatoire avec un niveau de qualité élevé. Le fait que récemment la presse s'est fait l'écho d'incidents graves dans un hôpital suite à

\section{«La constitution et la certification de tels centres tous les cinq ans a pour but de poursuivre l'optimisation de la médecine vasculaire en milieu hospitalier.»}

Enfin, au centre du vaisseau USSMV, les trois spécialistes se soutiennent réciproquement dans leur formation continue. Divers critères de formation continue se recoupent dans les trois spécialités angiologie, chirurgie vasculaire et radiologie. L'aide est alors apportée de manière collégiale, en toute simplicité et en suivant les aptitudes et les préférences des médecins qui peuvent aussi apprendre les contenus de formation continue de leurs collègues spécialistes.

\section{Perspectives}

Le centre du vaisseau USSMV donne priorité à la collaboration interdisciplinaire en bonne intelligence pour le bien du patient souffrant de maladie vasculaire complexe. La constitution et la certification de tels centres tous les cinq ans a pour but de poursuivre l'optimisation de la médecine vasculaire en milieu hospitalier. Elle va de pair avec la médecine vascu- une mauvaise collaboration interdisciplinaire nous renforce dans notre conviction que la qualité des processus avec une interdisciplinarité bien rodée prime sur les critères de jugement, voire en est la condition nécessaire. Des publications internationales sur la qualité donnent toujours plus souvent priorité à la qualité de la structure et des processus. Qui connaît le quotidien médical sait que la lutte pour les compétences, les frictions en matière de délimitation et d'autres heurts inutiles entre les disciplines influencent durablement la qualité des résultats et nuisent au patient. Ils coûtent également cher et empoisonnent l'ambiance de travail. Le centre du vaisseau USSMV suit l'idée de Jeremias Gotthelf selon laquelle «doit débuter dans le processus et l'esprit de corps et d'assistance ce dont le résultat est destiné à briller». La voie choisie par la Suisse pour la médecine vasculaire est trilatérale, pour le bien du patient et des collaborateurs satisfaits et motivés. 